\title{
Metamaterial-Inspired Multichannel Thin-Film Sensor
}

\author{
Withawat Withayachumnankul, Member, IEEE, Kata Jaruwongrungsee, Christophe Fumeaux, Senior Member, IEEE, \\ and Derek Abbott, Fellow, IEEE
}

\begin{abstract}
A multichannel thin-film sensor is implemented from a set of microstrip-coupled split-ring resonators (SRRs) with different dimensions. Each SRR exhibits a unique high-Q resonance that is sensitive to the presence of a sample in a particular area. Hence, this SRR-based sensor can function (i) to detect different samples simultaneously to increase the throughput or (ii) to characterise nominally identical samples at multiple frequencies to increase the sensor selectivity. In addition, the sensitivity of this SRRbased sensor is optimized through strategic design of the resonator shape to produce a strong confined electric field at each sensing region. The design principle is validated with simulation and measurement. Owing to the optimized design, sensing a low-permittivity film with a thickness as small as one thousandth of the operating wavelength is achievable.
\end{abstract}

Index Terms-Metamaterial, multichannel sensors, split-ring resonator.

\section{INTRODUCTION}

$\mathbf{S}$ PLIT-RING RESONATORs (SRRs) are among many fundamental building blocks for metamaterials that can collectively provide customizable values of the permittivity and/or permeability. A SRR is typically made of one or two concentric subwavelength metallic rings (namely single or double SRR, respectively) with a narrow split in each ring [1]. In response to electromagnetic excitation, it exhibits a strong magnetic resonance whose frequency is determined by its dimensions, geometry, and constituent materials. On resonance, the ring develops an intense and localised electric field at the narrow split. The sensitivity of the resonance frequency to constituent materials, together with the field localization, subwavelength ring size, and high-Q resonance, makes SRRs ideal for thin-film sensing.

Manuscript received July 26, 2011; accepted October 17, 2011. Date of publication October 27, 2011; date of current version April 13, 2012. This was supported by the Australian Research Council Discovery Projects funding scheme under Project DP1095151. The associate editor coordinating the review of this manuscript and approving it for publication was Prof. Gerald Gerlach.

W. Withayachumnankul is with the School of Electrical and Electronic Engineering, The University of Adelaide, Adelaide, SA 5005, Australia, and also with the School of Electronic Engineering, Faculty of Engineering, King Mongkut's Institute of Technology Ladkrabang, Bangkok 10520, Thailand (e-mail: withawat@eleceng.adelaide.edu.au).

K. Jaruwongrungsee is with the School of Electronic Engineering, Faculty of Engineering, King Mongkut's Institute of Technology Ladkrabang, Bangkok 10520, Thailand, and also with the Nanoelectronics and MEMS Laboratory, National Electronics and Computer Technology Center, Pathumthani 12120, Thailand (e-mail: kata.jaruwongrungsee@ nector.or.th).

C. Fumeaux and D. Abbott are with the School of Electrical and Electronic Engineering, The University of Adelaide, Adelaide, SA 5005, Australia (e-mail: cfumeaux@eleceng.adelaide.edu.au; dabbott@eleceng.adelaide.edu.au).

Color versions of one or more of the figures in this paper are available online at http://ieeexplore.ieee.org.

Digital Object Identifier 10.1109/JSEN.2011.2173762
Owing to these properties, SRRs have recently been implemented for biosensors in different configurations. Planar arrays of SRRs were employed for thin-film sensing [2]-[8]. However, those sensors require a relatively large amount of the sample to uniformly cover an array with tens to hundreds of SRRs during the measurement. The constraints of the sample size and uniformity can be eliminated by using either microstrip-coupled SRRs [9]-[11] or waveguide-loaded SRRs [12]. Although both microstrip and waveguide arrangements can be used for thin-film sensing, electromagnetic coupling is much stronger in the former case, resulting in its higher immunity against noise.

For the microstrip-coupling configuration, an SRR is positioned in a close proximity to a microstrip transmission line, which builds up a magnetic field around itself in a quasi-TEM wave propagation. This oscillating magnetic field induces circulating current in the SRR loop. In the quasi-static limit, an SRR can be approximated by an inductor and a capacitor in the form of a series $L C$ resonant circuit. Specifically, the ring forms the inductor, and the split forms the capacitor. The resonance takes place in the SRR when the electric energy stored in the capacitor is balanced with the magnetic energy stored in the inductor. Loading a sample onto the SRR surface alters the total capacitance of the structure and hence results in a detectable change in the resonance frequency.

This article presents a thin-film sensor based on microstripcoupled SRRs. Two significant improvements over previous designs are reported. First, the proposed sensor can detect the presence of multiple samples simultaneously. This function can be beneficial when a considerable number of samples have to be tested. Furthermore, since the sensor can characterize a nominally identical sample at multiple frequencies, the selectivity can be expected in sensing dispersive materials. The other improvement involves the sensitivity of the sensor. By creating a strong and localized field enhancement in the sensing region, a high sensitivity can be achieved.

\section{DESIGN AND FABRICATION}

In earlier designs, several double SRRs [9]-[11] or a single SRR with two splits [12] have been used for thin-film sensing. In such structures, several distributed capacitors collectively determine the resonance frequency, and a considerable amount of sample is required to alter the electrical properties all of these capacitors. If the sample partially covers the structure, the unaffected capacitors reduce the sensitivity in the sensing region as the capacitive change is then not as pronounced. In the present design, each resonance is caused by a single SRR with only one split, as shown in Fig. 1(a), and consequently, only one distributed capacitor is present. A change in this capacitor by 


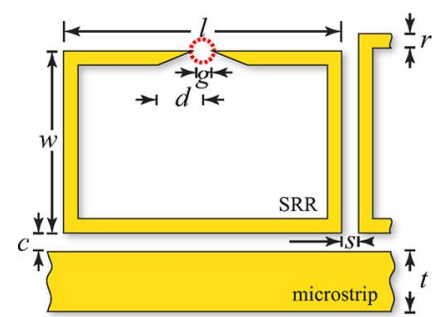

(a)

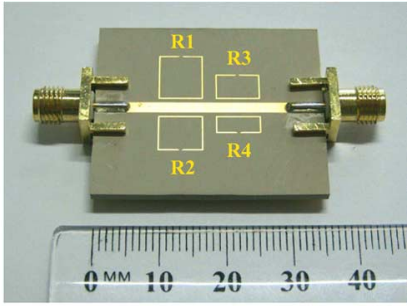

(b)
Fig. 1 (a) Diagram (not to scale) for each SRR, placed alongside a microstrip line, in the multichannel sensor. The sensing area is indicated by the red dotted circle. (b) Fabricated four-channel sensor containing four SRRs.

sample loading in the small split region has a direct and large impact on the resonance frequency.

Fig. 1(a) also depicts some other important features of the implemented resonators. Each SRR has a rectangular shape to maximize coupling to the microstrip transmission line. This leads to a stronger resonance that is robust to measurement uncertainties. At each SRR split, sharp tips are adopted to concentrate the electric field to a small spot [12]. In terms of a series $L C$ circuit, the tapered shape of the tips decreases the capacitance at the split, and hence increases the resonance Q-factor.

In order to perform multichannel sensing, several optimized SRRs with different sizes are placed along a microstrip line. Thus, the sensor exhibits multiple resonances, each of which represents a single channel governed by an individual SRR. Each resonance is sensitive to the presence of a sample deposited at the narrow split of the corresponding SRR. Since every resonator possesses a high- $Q$ resonance at a unique frequency, mutual interaction among the resonators is minimal. Therefore, several SRRs can be placed densely without compromising the performance.

Fig. 1(b) shows the proposed sensor fabricated by using standard photolithography and chemical etching. This particular four-channel sensor is realized from four different SRRs positioned along a $50-\Omega$ microstrip line. All of the resonators share the same dimensions excepting the width, $w$, that equals 8,6 , 4.5 , and $3 \mathrm{~mm}$ for R1, R2, R3, and R4, respectively. This causes a difference in the loop inductance and hence in the resonance frequency among the SRRs. The other dimensions common to all of the SRRs are as follows: $l=7 \mathrm{~mm}, g=0.15 \mathrm{~mm}$, $d=0.525 \mathrm{~mm}, r=0.2 \mathrm{~mm}, c=0.65 \mathrm{~mm}, s=2 \mathrm{~mm}$, and $t=1.7 \mathrm{~mm}$. The metal for the SRRs, microstrip, and ground plane is copper with a thickness of $35 \mu \mathrm{m}$, coated with gold to prevent oxidization. The substrate is an RT/duroid 6010.2LM high-frequency laminate (ceramic-PTFE composite) with a thickness of $1.90 \mathrm{~mm}$, a relative permittivity of 10.2, and a loss tangent of 0.0023 . This sensor specification yields four high-Q resonances in the microwave $\mathrm{S}$ band.

\section{EXPERIMENT AND RESULTS}

\section{A. Sensor Characteristics}

During the experiment, the sensor is connected to a vector network analyzer, Agilent Technologies N5230A, and the transmission parameter $S_{21}$ is registered at room temperature in the range between $1.5-3.5 \mathrm{GHz}$ with a resolution of $3 \mathrm{MHz}$.

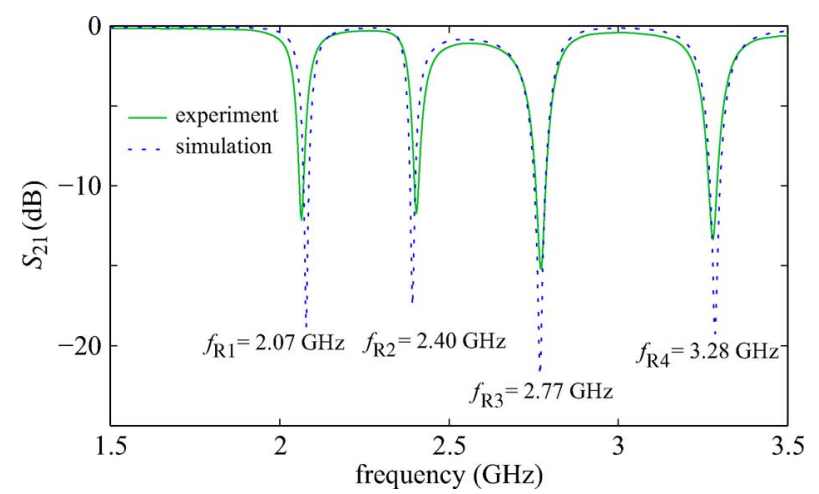

Fig. 2. Transmission profiles from simulation and experiment with no sample loading. Each resonance, i.e., channel, corresponds to an individual SRR. The indicated resonance frequencies are extracted from the experimental results.

The numerical simulation is carried out with a commercial full-wave electromagnetic solver, CST Microwave Studio, based on the Finite-Integration Technique (FIT). The gap size $g$ in each ring resonator is retrospectively slightly adjusted in the simulation to reflect the fabrication imperfections, as observed under a microscope.

The measured and simulated transmission profiles of the sensor without any sample are shown in Fig. 2 with small discrepancies caused by fabrication tolerances and limits in simulation accuracy. The results show four non-overlapping resonances corresponding to the four distinct SRRs. The lowest resonance $f_{\mathrm{R} 1}$ is associated with $\mathrm{R} 1$, which has the largest inductance, the second lowest resonance $f_{\mathrm{R} 2}$ with $\mathrm{R} 2$, and so on. No change in the resonances is observed during 5 hours of measurement, demonstrating the thermal stability of the sensor.

Further insight can be obtained from the simulation, as shown in Fig. 3, where the electric-field distribution on the sensor surface is calculated for every channel. At each resonance frequency, only the relevant SRR establishes a strong and highly confined electric field around the capacitive split, and no field enhancement is observed elsewhere. The field enhancement in any active ring is as high as $380 \mathrm{kV} / \mathrm{m}$, given the input power of only $1 \mathrm{~W}$. These calculated field distributions confirm the extreme field confinement achieved with the implemented SRRs, which is a basis for high-performance sensing.

\section{B. Multichannel sensing}

In the next experiment, ethanol is used as a sample under test by the multichannel sensor. At $3 \mathrm{GHz}$, ethanol has a relative permittivity and loss tangent of 6.0 and 0.96 , respectively [13]. In each measurement, $5 \mu \mathrm{L}$ of ethanol is dropped by using a highprecision pipette onto the split of a selected SRR channel, and the sensor's response is observed. The transmission results are shown in Fig. 4. The observed resonance shifting and damping indicate the presence of the sample in the selected channel. By comparing the resonance frequencies of the loaded sensor in Fig. 4 with the reference values in Fig. 2, it is clear that non-active SRR channels show no detectable change in the resonance frequency. Hence, it is clear that the four sensing channels can operate independently.

Each SRR can be approximated by a series $L C$ circuit with a resonance frequency $f_{0}=1 /(2 \pi \sqrt{L C})$, where $L$ denotes 


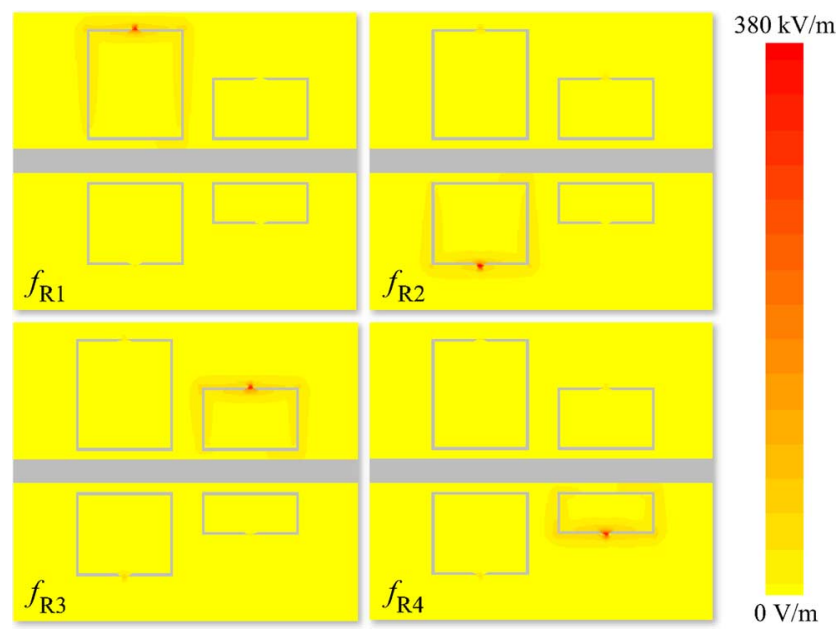

Fig. 3. Simulated electrical-field amplitude distribution on the sensor surface at four resonance frequencies. The peak input power supplied to the sensor is $1 \mathrm{~W}$. The color scale is linear.

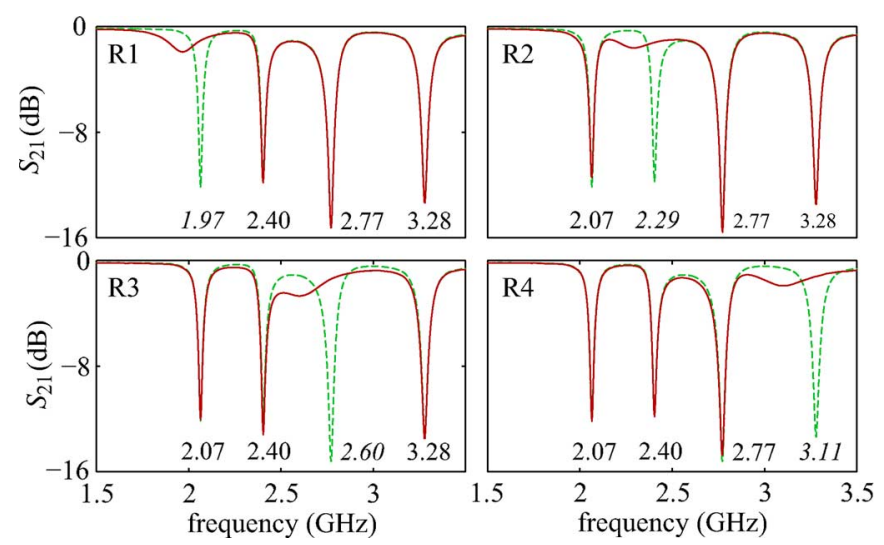

Fig. 4. Transmission profiles for unloaded (dotted lines) and loaded (solid lines) SRRs. Each subfigure represents a comparison of sample loading at an individual SRR channel. The specified numbers are the resonance frequencies in $\mathrm{GHz}$ of the loaded sensor, and the italization indicates shifted resonances.

the ring inductance and $C$ denotes the split capacitance. The sensitivity of the resonance frequency to the split capacitance can be expressed as [14]

$$
S_{C}^{f_{0}}=\frac{C}{f_{0}} \frac{\partial f_{0}}{\partial C}=-0.5 .
$$

It can be deduced from this equation that if the relative change in the capacitance formed by the split, $\Delta C / C$, is constant, the relative change in the resonance frequency, $\Delta f / f_{0}$, is fixed, regardless of the ring inductance. Nonetheless, the relative change in the resonance frequency for the active channel varies between $-4.6 \%$ and $-6.1 \%$ among the four channels, despite a fixed volume of sample. This small variation is presumably caused by manual sample deposition, which results in a slight difference in the sample location. This uncertainty can be readily overcome via an automated process.

The sample volume used in the experiment is considerably small. Nevertheless, additional simulations suggest that only one nanolitre of ethanol, i.e., a $0.1-\mathrm{mm}$ cube, is unambiguously detectable with a frequency shift of $15 \mathrm{MHz}$. In other words, the ratio between the operating wavelength and the film thickness can be as large as three orders of magnitude.

\section{CONCLUSION AND FUTURE WORK}

In conclusion, this article presents a thin-film sensor with a series of optimized SRRs as sensing elements. The fabricated sensor successfully performs multichannel detection with an enhanced sensitivity. Scaling down the structure to operate at higher frequencies further reduces the minimum amount of detectable sample. More channels can be added to the sensor provided that resulting resonances are sparse enough to avoid mutual coupling. The resonance of each channel can be positioned at an abritary frequency where unique dielectric features of the sample are expected. The realization can be used for on-site disposable sensors, which allow sensing with either high selectivity or high throughput.

\section{ACKNOWLEDGMENT}

The authors acknowledge P. Simcik and H. Ho for their technical assistance. The laminates used in the experiment were supplied by the Rogers Corporation.

\section{REFERENCES}

[1] J. B. Pendry, A. J. Holden, D. J. Robbins, and W. J. Stewart, "Magnetism from conductors and enhanced nonlinear phenomena," IEEE Trans. Microw. Theory Tech., vol. 47, no. 11, pp. 2075-2084, Nov. 1999.

[2] C. Debus and P. H. Bolivar, "Frequency selective surfaces for high sensitivity terahertz sensing," Appl. Phys. Lett., vol. 91, 2007, Art. ID 184102.

[3] T. Driscoll, G. O. Andreev, D. N. Basov, S. Palit, S. Y. Cho, N. M. Jokerst, and D. R. Smith, "Tuned permeability in terahertz split-ring resonators for devices and sensors," Appl. Phys. Lett., vol. 91, 2007, Art. ID 062511.

[4] Y.-T. Chang, Y.-C. Lai, C.-T. Li, C.-K. Chen, and T.-J. Yen, "A multi-functional plasmonic biosensor," Opt. Exp., vol. 18, no. 9, pp. 9561-9569, 2010.

[5] S.-Y. Chiam, R. Singh, J. Gu, J. Han, W. Zhang, and A. A. Bettiol, "Increased frequency shifts in high aspect ratio terahertz split ring resonators," Appl. Phys. Lett., vol. 94, no. 6, 2009, Art. ID 064102.

[6] J. F. O'Hara, R. Singh, I. Brener, E. Smirnova, J. Han, A. J. Taylor, and W. Zhang, "Thin-Film sensing with planar terahertz metamaterials: Sensitivity and limitations," Opt. Exp., vol. 16, no. 3, pp. 1786-1795, 2008.

[7] J. A. Gordon, C. L. Holloway, J. Booth, S. Kim, Y. Wang, J. BakerJarvis, and D. R. Novotny, "Fluid interactions with metafilms/metasurfaces for tuning, sensing, and microwave-assisted chemical processes," Phys. Rev. B: Condens. Matter, vol. 83, 2011, Art. ID 205130.

[8] H. Tao, L. Chieffo, M. A. Brenckle, S. M. Siebert, M. Liu, A. C. Strikwerda, K. Fan, D. L. Kaplan, X. Zhang, R. D. Averitt, and F. G. Omenetto, "Metamaterials on paper as a sensing platform," $A d v$. Mater., vol. 23, no. 28, pp. 3197-3201, 2011.

[9] H.-J. Lee and J.-G. Yook, "Biosensing using split-ring resonators at microwave regime," Appl. Phys. Lett., vol. 92, 2008, Art. ID 254103.

[10] H.-J. Lee, H.-S. Lee, K.-H. Yoo, and J.-G. Yook, "On the possiblity of biosensors based on split ring resonators," in Proc. 38th Eur. Microw. Conf., 2008, pp. 1222-1225.

[11] H.-J. Lee, H.-S. Lee, K.-H. Yoo, and J.-G. Yook, "DNA sensing based on single element planar double split-ring resonator," in IEEE MTT-S Int. Microw. Symp. Dig., 2009, pp. 1685-1688.

[12] I. A. I. Al-Naib, C. Jansen, and M. Koch, "Thin-film sensing with planar asymmetric metamaterial resonators," Appl. Phys. Lett., vol. 93, 2008, Art. ID 083507.

[13] R. E. Mudgett, D. I. C. Wang, and S. A. Goldblith, "Prediction of dielectric properties in oil-water and alcohol-water mixtures at 3000 $\mathrm{MHz}, 25^{\circ} \mathrm{C}$ based on pure component properties," J. Food Sci., vol. 39, pp. 632-635, 1974

[14] W. Withayachumnankul, C. Fumeaux, and D. Abbott, "Planar array of electric-LC resonators with broadband tunability," IEEE Antennas Wire. Propag. Lett., vol. 10, pp. 577-580, 2011. 


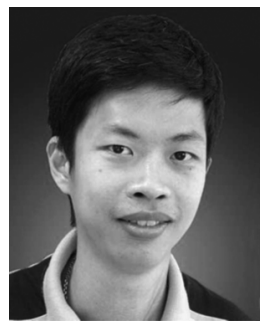

Withawat Withayachumnankul (M'11) received the B.Eng. and M.Eng degrees in electronic engineering from King Mongkut's Institute of Technology at Ladkrabang (KMITL), Bangkok, Thailand, in 2001 and 2003, respectively, and the $\mathrm{Ph} . \mathrm{D}$. degree in electrical engineering from the University of Adelaide, Adelaide, Australia, in 2010.

He served as a Lecturer at KMITL with the Faculty of Engineering. Currently, he holds an ARC Australian postdoctoral fellowship with the University of Adelaide. His research interests include metamaterials and terahertz technology.

Dr Withayachumnankul received an Australian Endeavour International Postgraduate Research Scholarship (2006), University of Adelaide Scholarship for Postgraduate Research (2006), IEEE/LEOS Graduate Student Fellowship (2008), SPIE Scholarship in Optical Science and Engineering (2008), award for the poster presentation at the SPIE Symposium on Microelectronics, MEMS, and Nanotechnology, Canberra, Australia, (2007), contingency travel grant of the SPIE, (2007), and CSIRO travel grant for the Nanomaterial-Based Sensors for Biomedical Applications symposium (2011).

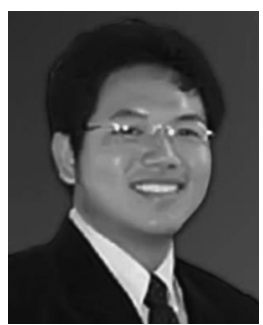

Kata Jaruwongrungsee received the B.Eng. and M.Eng. degrees in electronics engineering from King Mongkut's Institute of Technology Ladkrabang (KMITL), Bangkok, Thailand, in 2003 and 2005 , respectively, where he is currently working toward the Ph.D. degree in electronics engineering.

Since 2006, he has been with the Nanoelectronics and MEMS Laboratory, National Electronics and Computer Technology Center (NECTEC), Thailand, as an Assistant Researcher. His research is mainly focused on QCM-based sensor technology and its

applications.

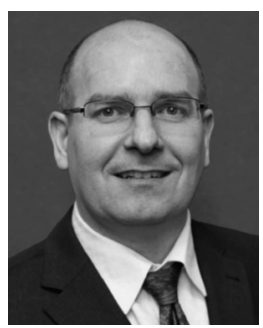

Christophe Fumeaux (M'03-SM'09) received the Diploma and Ph.D. degrees in physics from the ETH Zürich, Switzerland, in 1992 and 1997, respectively.

From 1998 to 2000, he was a Postdoctoral Researcher with the School of Optics, University of Central Florida, Orlando. In 2000, he joined the Swiss Federal Office of Metrology, Bern, Switzerland, as a Scientific Staff Member. From 2001 to 2008, he was a Research Associate and Group Leader with the Laboratory for Electromagnetic Fields and Microwave Electronics (IFH), ETH Züurich. During Fall 2005, he was a Visiting Scientist with the Laboratory of Sciences and Materials for Electronics and Automation (LASMEA), University Blaise Pascal, Clermont-Ferrand, France. In 2008, he joined the School of Electrical and Electronic Engineering, The University of Adelaide, Adelaide, Australia, as an Associate Professor. Since 2011, he is a Future Fellow of the Australian Research Council. His current main research interest concerns computational electromagnetics, antenna engineering, terahertz technology, and the modeling of optical microstructures/nanostructures.

Dr Fumeaux is an Associate Editor for the IEEE TRANSACTIONS ON Microwave THEORY AND TeChNiQues. He was the chairman of the IEEE Swiss Joint Chapter on Microwave Theory and Techniques (MTT-S), Antennas and Propagation (AP-S), and Electromagnetic Compatibility (EMC) from 2006 to 2008. Since 2010, he has been the chairman of the IEEE South Australia AP-S/MTT-S Chapter. He was the recipient of the ETH Silver Medal of Excellence for his doctoral dissertation. He was the corecipient of the 2004 Outstanding Paper Award of the Applied Computational Electromagnetics Society (ACES).

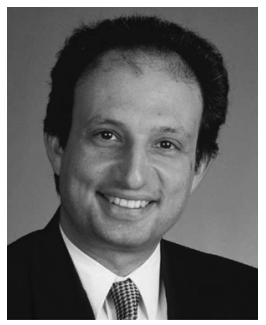

Derek Abbott (M'85-SM'99-F'05) was born on May 3, 1960, in South Kensington, London, U.K. $\mathrm{He}$ received the B.Sc. (Hons) degree in physics from Loughborough University of Technology, Leicestershire, U.K., in 1982, and the Ph.D. degree (with commendation) in electrical and electronic engineering from The University of Adelaide, Adelaide, Australia, in 1995 under Kamran Eshraghian and Bruce R. Davis.

He has led a number of research programs in the imaging arena, ranging from the optical to infrared to millimeter wave to T-ray (terahertz gap) regimes. From 1978 to 1986, he was with GEC Hirst Research Centre, London, U.K., working in the area of visible and infrared image sensors. His expertise also spans VLSI design, optoelectronics, device physics, and noise; where he has worked with nMOS, CMOS, SOS, CCD, GaAs, and vacuum microelectronic technologies. On migration to Australia, he worked for Austek Microsystems, Technology Park, South Australia, in 1986. Since 1987, he has been with The University of Adelaide, where he is presently a full Professor with the School of Electrical and Electronic Engineering. He has appeared on national and international television and radio and has also received scientific reportage in New Scientist, The Sciences, Scientific American, Nature, The New York Times, and Sciences et Avenir. He holds over 300 publications/patents and has been an invited speaker at over 80 institutions, including Princeton, NJ; MIT, MA; Santa Fe Institute, NM; Los Alamos National Laboratories, NM; Cambridge, UK; and EPFL, Lausanne, Switzerland. He coauthored the book Stochastic Resonance (Cambridge University Press, 2008) and coedited the book Quantum Aspects of Life (Imperial College Press, 2008).

Prof Abbott is a Fellow of the Institute of Physics (IOP), with honorary life membership. He won the GEC Bursary (1977), the Stephen Cole the Elder Prize (1998), the E.R.H. Tiekink Memorial Award (2002), SPIE Scholarship Award for Optical Engineering and Science (2003), the South Australian Tall Poppy Award for Science (2004) and the Premier's SA Great Award in Science and Technology for outstanding contributions to South Australia (2004). He has served as an editor and/or guest editor for a number of journals including the IEEE Journal of SOLID-STATE CIRCUITS, Chaos, Smart Structures and Materials, Journal of Optics B, Microelectronics Journal, and Fluctuation Noise Letters and is currently on the Editorial Boards of the PROCEEDINGS OF THE IEEE and IEEE PHOTONICS. He has served on a number of IEEE technical program committees, including the IEEE APCCS and the IEEE GaAs IC Symposium. 